\title{
RESEARCH ON ATTITUDE CONTROL ALGORITHM OF QUADROTOR BASED ON PID
}

\author{
Chenyu Hu ${ }^{1}$, Hui Yang ${ }^{2}$ \\ ${ }^{1}$ Student(PG), Department of Air Transportation, Shanghai University of Engineering Science, China \\ ${ }^{2}$ Lecturer, Department of Air Transportation, Shanghai University of Engineering Science, China
}

\begin{abstract}
Quadrotor is a multivariable, strong coupling of the owe drive system, it is very difficult to control its exact. The difficulty is that it is difficult to establish an accurate mathematical model for the quadrotor, and secondly, the control algorithm is used to control the quadrotor. According to the two problems, the mathematical model of the quadrotor is established according to the Newton Euler law. Although their have some intelligent control algorithm, but considering its practical application in complicated control method using mature PID control, simulation of the Simulink model is used to verify that the PID has good effect on the attitude control of quadrotor. In this paper, we mainly study the attitude control, so we did not design the controller of the three axis of the quadrotor.
\end{abstract}

Keywords: Quadrotor, Attitude Control, PID Control, Mathematical Model

\section{INTRODUCTION}

Quadrotor first appeared in 1920s, began as a military training purposes, and later due to the excellent performance in the military, it quickly attracted the attention of countries around the world. Compared with the conventional quadrotor, quadrotor has the advantages of simple structure, light weight, small volume, easy maintenance, low cost, flexibility, portability, simple operation and other advantages. So both in military and civil fields, the application prospect of quadrotor are very broad [1].

Quadrotor is a multi rotor quadrotor non coaxial vertical takeoff and landing[2], only by adjusting the four rotor speed to control the quadrotor flight attitude. The quadrotor with four symmetrical distribution of the rotor, thus can offset each rotor anti torque torque, so there is no need to make the tail, quadrotor has the advantages of simple structure [3]. The lift of the four rotors is more uniform than that of the single rotor, and the flight attitude is more stable than that of the single rotor [4]. Because the quadrotor has six degrees of freedom, but only four of the control of the motor speed, so it is a typical strong coupling, nonlinear, multi variable under the drive system [5]. In order to enhance the stability and safety of the quadrotor, an accurate mathematical model and an excellent control system are established. In this paper, the classical PID algorithm is used to control the quadrotor and Simulation on the Simulink, which has a good control effect.

\section{MATHEMATICAL MODEL ESTABLISHMENT}

Modeling of the quadrotor must solve the problem of the coordinate system, so the establishment of two coordinate system of the ground coordinate system $G$ and the body coordinate system $B$, as shown in figure 1 :

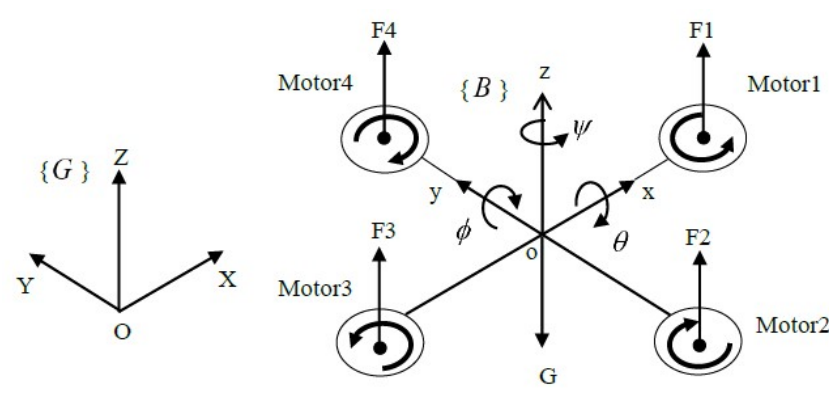

Fig -1: Structure Model of quadrotor

Usually the position and attitude of the quadrotor is defined in the ground coordinate system, and the sensor is the attitude 
and position information of the vehicle body coordinate system, so it is necessary to the body coordinate system to the ground coordinate system. Assuming the body roll angle $\alpha=[\theta, \phi, \psi]$, which $\theta$ is the roll angle, $\phi$ is the pitch angle, $\psi$ is the yaw angle, each angle is defined as shown in figure 2:

Roll angle $\theta$ : the coordinate system $o x y z$ around the $x$ axis, the $Y$ axis in the $o y z$ plane projection and the angle of the $y$ axis;

Pitch angle $\phi$ : the coordinate system $o x y z$ around the $y$ axis, the $Z$ axis in the ozx plane projection and the angle of the $z$ axis;

Yaw angle $\psi$ : the coordinate system $o x y z$ around the $z$ axis, the $X$ axis in the $o x y$ plane projection and the angle of the $x$ axis;

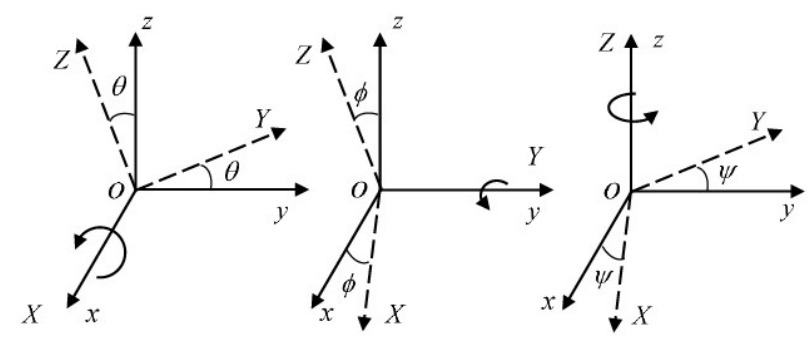

Fig -2:Schematic diagram of coordinate system transformation

The coordinate transformation matrix of each angle is obtained:

$R_{\theta}=\left[\begin{array}{ccc}1 & 0 & 0 \\ 0 & \cos \theta & -\sin \theta \\ 0 & \sin \theta & \cos \theta\end{array}\right]$

$R_{\phi}=\left[\begin{array}{ccc}\cos \phi & 0 & \sin \phi \\ 0 & 1 & 0 \\ -\sin \phi & 0 & \cos \phi\end{array}\right]$

$$
R_{\psi}=\left[\begin{array}{ccc}
\cos \psi & -\sin \psi & 0 \\
\sin \psi & \cos \psi & 0 \\
0 & 0 & 1
\end{array}\right]
$$

Finally get the whole 3D space coordinate transformation matrix:

$R=R_{\psi} \times R_{\phi} \times R_{\theta}=\left[\begin{array}{ccc}\cos \psi \cos \phi & \cos \psi \sin \phi \sin \theta-\sin \psi \cos \theta & \cos \psi \sin \phi \cos \theta+\sin \psi \sin \theta \\ \sin \psi \cos \phi & \sin \psi \sin \phi \sin \theta+\cos \psi \cos \theta & \sin \psi \sin \phi \cos \theta-\cos \psi \sin \theta \\ -\sin \phi & \cos \phi \sin \theta & \cos \phi \cos \theta\end{array}\right]$

Vector in the body coordinate system:

$$
A_{B}=\left[x_{B}, y_{B}, z_{B}\right]^{T}
$$

Is converted to the ground coordinate system:

$$
A_{G}=\left[x_{G}, y_{G}, z_{G}\right]^{T}=R \times A_{B}
$$

In order to establish the dynamics model of the quadrotor, the following assumptions are made to the quadrotor [6]:

1. Quadrotor for uniform and symmetrical rigid body;

2. The origin of the ground coordinate system is the geometric center of the quadrotor, and is coincident with the center of mass;

3. The body is affected by the air resistance can be ignored, regardless of the ground effect;

4. Four the lift of each rotor is proportional to the square of the rotor speed, and the torque of the rotor is proportional to the square of the rotor speed;

Based on Newton's second law and Euler's formula, the dynamics model equation of quadrotor is established [7]:

$$
F=m \frac{d V}{d t}, M=\frac{d H}{d t}
$$

$F$ is the sum of the external forces of the quadrotor, $m$ for the quality of the quadrotor, $V$ is the speed of the quadrotor, $M$ for the quadrotor by the sum of the torque, $H$ quadrotor relative to the ground coordinates of the moment of 
momentum.

Set a single propeller speed is $\Omega_{n}$,by the assumption of 4 to each rotor lift is $a \Omega_{n}^{2}$, where $a$ is the proportional coefficient. The total lift of the quadrotoris $U_{1}=a\left(\Omega_{1}^{2}+\Omega_{2}^{2}+\Omega_{3}^{2}+\Omega_{4}^{2}\right) \quad$, the force vector $F_{B}=\left(0,0, U_{1}\right)^{T}$ of the quadrotor in the body coordinate system $B$. By the formula (6) can get the ground coordinate system under the force vector is:

$F_{G}=\left[\begin{array}{l}F_{X} \\ F_{Y} \\ F_{Z}\end{array}\right]=R \times F_{B}=\left[\begin{array}{c}(\cos \psi \sin \phi \cos \theta+\sin \psi \sin \theta) U_{1} \\ (\sin \psi \sin \phi \cos \theta-\cos \psi \sin \theta) U_{1} \\ (\cos \phi \cos \theta) U_{1}\end{array}\right]$

The air drag coefficient of the three rotor quadrotoris $X, Y$ and $Z$ in the ground coordinate system. The air resistance coefficient is $K_{X}, K_{Y}, K_{Z}$, and $F=m \frac{d V}{d t}$, according to Newton's second law, the three axis linear equation of the ground coordinate system is established:

$\left[\begin{array}{c}\ddot{x} \\ \ddot{y} \\ \ddot{z}\end{array}\right]=\left[\begin{array}{c}\frac{F_{X}-K_{X} \dot{x}}{m} \\ \frac{F_{Y}-K_{Y} \dot{y}}{m} \\ \frac{F_{Z}-K_{Z} \dot{z}-m g}{m}\end{array}\right]=\left[\begin{array}{c}\frac{(\cos \psi \sin \phi \cos \theta+\sin \psi \sin \theta) U_{1}-K_{X} \dot{x}}{m} \\ \frac{(\sin \psi \sin \phi \cos \theta-\cos \psi \sin \theta) U_{1}-K_{Y} \dot{y}}{m} \\ \frac{(\cos \phi \cos \theta) U_{1}-K_{Z} \dot{z}}{m}-g\end{array}\right]$

(9)

Due to the role of force is mutual, the air will also have a reaction force on the rotor, so the yaw moment is so generated, the torque generated by each rotor is $W_{n}=b \Omega_{n}{ }^{2}$, where $b$ is the reverse torque coefficient. $l$ for the rotor center to the center of the quadrotor to the distance, $U_{2}$ for the rolling moment, $U_{3}$ for the pitching moment, $U_{4}$ for the yaw moment, there are:

$$
\left[\begin{array}{l}
U_{2} \\
U_{3} \\
U_{4}
\end{array}\right]=\left[\begin{array}{c}
l\left(F_{4}-F_{2}\right) \\
l\left(F_{3}-F_{1}\right) \\
W_{4}+W_{2}-W_{3}-W_{1}
\end{array}\right]=\left[\begin{array}{c}
l a\left(\Omega_{4}^{2}-\Omega_{2}^{2}\right) \\
l a\left(\Omega_{3}^{2}-\Omega_{1}^{2}\right) \\
b\left(\Omega_{4}^{2}+\Omega_{2}^{2}-\Omega_{3}^{2}-\Omega_{1}^{2}\right)
\end{array}\right]
$$

Because of the assumption 1, the inertia matrix can be defined as the diagonal matrix $J$, and the three axis angular motion equations can be obtained according to the Euler dynamic equation of rigid body fixed point rotation:

$$
\left[\begin{array}{c}
\ddot{\theta} \\
\ddot{\phi} \\
\ddot{\psi}
\end{array}\right]=\left[\begin{array}{c}
\frac{U_{2}+\left(J_{y}-J_{z}\right) \dot{\phi} \dot{\psi}}{J_{x}} \\
\frac{U_{3}+\left(J_{z}-J_{x}\right) \dot{\psi} \dot{\theta}}{J_{y}} \\
\frac{U_{4}+\left(J_{x}-J_{y}\right) \dot{\theta} \dot{\phi}}{J_{z}}
\end{array}\right]
$$

As the quadrotor flying in the wind or in indoor environment, so we can ignore the resistance coefficient are studied, so a simplified mathematical model of the final as follows:

$$
\left[\begin{array}{c}
\ddot{x} \\
\ddot{y} \\
\ddot{z}
\end{array}\right]=\left[\begin{array}{c}
(\cos \psi \sin \phi \cos \theta+\sin \psi \sin \theta) \frac{U_{1}}{m} \\
(\sin \psi \sin \phi \cos \theta-\cos \psi \sin \theta) \frac{U_{1}}{m} \\
(\cos \phi \cos \theta) \frac{U_{1}}{m}-g
\end{array}\right]
$$

\section{CONTROLLER DESIGN}

In this paper, the design of the controller based on the 
classical PID control method, the control law of the system is designed using closed loop control. The principle of the PID controller is to make the system's output value and expected value to do the difference, and the error signal proportional, integral, differential calculation, the three parts of the sum to get the final control signal [8]. Figure 3 for the PID control schematic.

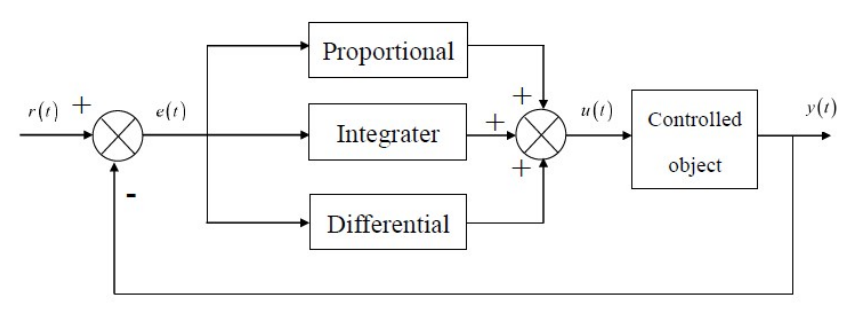

Fig -3: PID control schematic

Equations (12) and (13) establish the three-axis dynamic model and the three-point attitude dynamics model of the quadrotor respectively. The whole model can be regarded as two independent systems: linear motion and angular motion. From the formulas (12) and (13), it can be seen that the attitude change of the aircraft directly affects the linear motion of the quadrotor, while the linear motion does not cause the attitude change. As the focus of this paper is attitude control simulation, we do not consider the three-axis linear control simulation, only three attitude control simulation. The authors refer to the relevant literature to get the quadrotor parameters as followsTable 1:

Table -1: parameters of quadrotor

\begin{tabular}{|c|c|c|c|}
\hline Parameter & Description & Value & Units \\
\hline$m$ & Mass & 0.504 & $\mathrm{~kg}$ \\
\hline$l$ & Distance & 0.25 & $\mathrm{~m}$ \\
\hline$a$ & $\begin{array}{c}\text { Proportionality } \\
\text { Constant }\end{array}$ & $3.5 \times 10^{-6}$ & $\mathrm{~N} \cdot \mathrm{S}^{2} \cdot \mathrm{rad}^{-2}$ \\
\hline$b$ & $\begin{array}{c}\text { Proportionality } \\
\text { Constant }\end{array}$ & $1.1 \times 10^{-6}$ & $\mathrm{~N} \cdot \mathrm{S}^{2} \cdot \mathrm{rad}^{-2}$ \\
\hline$J_{x}$ & $x$ Inertia & $5.7 \times 10^{-3}$ & $\mathrm{~kg} \cdot \mathrm{m}^{2}$ \\
\hline$J_{y}$ & $y$ Inertia & $5.7 \times 10^{-3}$ & $\mathrm{~kg} \cdot \mathrm{m}^{2}$ \\
\hline$J_{z}$ & $z$ Inertia & $9.2 \times 10^{-3}$ & $\mathrm{~kg} \cdot \mathrm{m}^{2}$ \\
\hline
\end{tabular}

Equations (13) the introduction of the control variable $U$ decomposes the complex nonlinear coupling model into three independent control channels. The 4 attitude PID circuit structure, according to figure 4 to build the Simulink model and simulation, after debugging the attitude angles, the PID parameter is shown in Table 2, followed by the pitch angle, roll angle and yaw angle, the attitude controller design step response is shown in Figure 5.

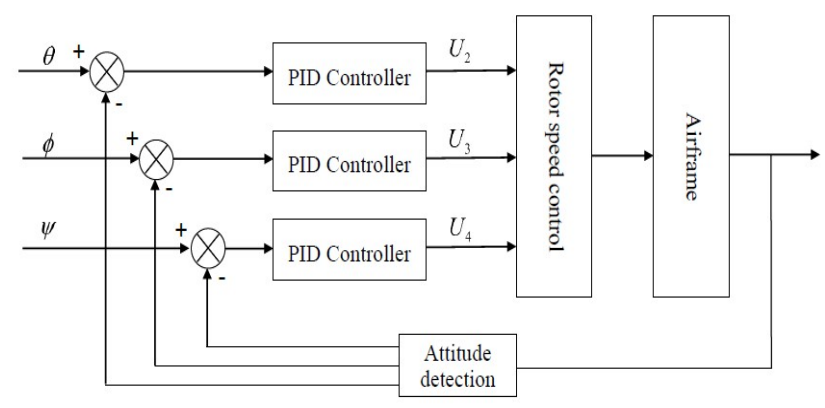

Fig -4: The structure of attitude PID circuit

Table -2: Parameters of each attitude angle

\begin{tabular}{|c|c|c|c|}
\hline$N$ & $K_{P}$ & $K_{I}$ & $K_{D}$ \\
\hline 1 & 2.8 & 8.9 & 0.3 \\
\hline 2 & 2.8 & 8.9 & 0.3 \\
\hline 3 & 2 & 5 & 0.26 \\
\hline
\end{tabular}
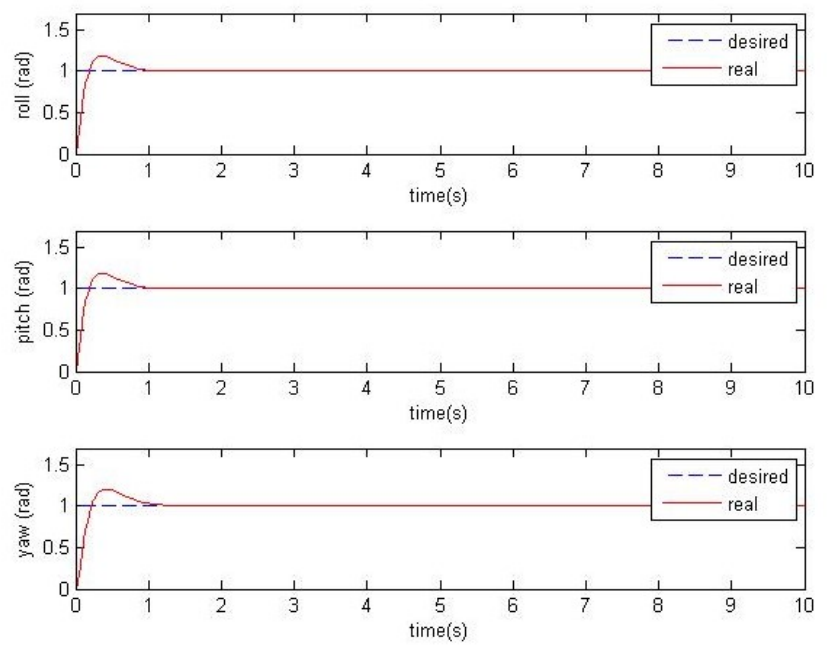

Fig -5: Step response of each attitude angle

The simulation results show that the PID has small control overshoot, almost no steady state error, shorter adjustment 
time and fast response speed, which shows that the PID has good control effect on the attitude of the quadrotor.

\section{CONCLUSION}

Quadrotor system is a multi variable and strong coupling, the precise control is very difficult, the modeling of the quadrotor dynamics model, control algorithm adopts mature PID control, using Simulink to build model and verify the effectiveness of PID for the four rotary wing quadrotor attitude control.

The study of the quadrotor is a multidisciplinary subject, and the knowledge involved is very wide. In view of the limited time, this paper only controls the attitude angle in flight control, and does not study the three-axis motion control. The future will be the attitude angle control and three-axis directional motion control to complete the control of the entire quadrotor control.

\section{REFERENCES}

[1]. Hu Jintian, Lin Lin. Research on the control algorithm of quadrotor based on PID neural network [J]. automation and information engineering, 2015,01:18-22.

[2]. Nie Bowen, Ma Hongxu, Wang Jian, Wang Jianwen. Research status and key technologies of Micro Quadrotor [J].electro optic and control, 2007,06:113-117.

[3]. tree leaf ball, Jennings. PID quadrotor attitude control system of $[\mathrm{J}]$.computer and modernization based on 2015,05:117-120.

[4]. Li Yuntang, Li Jun. Dynamics modeling and PID control of quadrotor [J]. Journal of Liaoning Technical University (NATURAL SCIENCE EDITION), 2012,01:114-117.

[5]. Li Yannong, Li Tinglan, Jiang Yi, van Jialu. RBF neural network adaptive PID control of quadrotor [J].control engineering based on 2016,03:378-382.

[6]. Ly Dat Minh. Modeling and control of quadrotor MAV using visionbasedmeasurement[J]. IEEE Trans. Circuits Syst., 2010, 33(4): 70-77.

[7]. Deng. Inertial technology [M]. Harbin Institute of Technology press, 2006:57-60.

[8]. Dai Qing, Li Hangyu. Attitude calculation and control of the quadrotor $[\mathrm{J}]$. science and technology information, 2015,35:33-34. 